\title{
Erratum to: The Frölicher spectral sequence can be arbitrarily non-degenerate
}

\author{
Laura Bigalke · Sönke Rollenske
}

Published online: 22 January 2014

(C) Springer-Verlag Berlin Heidelberg 2014

Erratum to: Math. Ann. (2008) 341:623-628

DOI 10.1007/s00208-007-0206-z

\section{Introduction}

In the original publication the second author constructed for $n \geq 2$ a series of compact complex manifolds $X_{n}$ and an element $\left[\beta_{1}\right]$ in the $E_{n}$-term of the Frölicher spectral sequence claiming that $d_{n}\left(\left[\beta_{1}\right]\right) \neq 0$ (Lemma 2 in loc.cit.). This claim is incorrect: we explain in Remark 2 that on the contrary $\beta_{1}$ induces a class in $E_{\infty}$.

However, the main result of the original publication remains true (up to a change in the dimension of the examples).

Theorem 1 For every $n \geq 2$ there exist a complex $4 n-2$-dimensional compact complex manifold $X_{n}$ such that the Frölicher spectral sequence does not degenerate at the $E_{n}$ term, i.e., $d_{n} \neq 0$.

The method of construction has remained the same, but we needed to introduce some extra counting variables.

We believe that in every dimension there are examples of nilmanifolds with leftinvariant complex structure where the maximal possible non-degeneracy occurs, but the structure equations might be quite complicated.

The online version of the original article can be found under doi:10.1007/s00208-007-0206-z.

L. Bigalke $\cdot$ S. Rollenske $(\bowtie)$

Fakultät für Mathematik, Universtät Bielefeld, Universitätsstr. 25,

33615 Bielefeld, Germany

e-mail: rollenske@math.uni-bielefeld.de 


\section{Construction of the example}

Consider the space $G_{n}:=\mathbb{C}^{4 n-2}$ with coordinates

$$
x_{1}, \ldots, x_{n-1}, y_{1}, \ldots, y_{n}, z_{1}, \ldots, z_{n-1}, w_{1}, \ldots, w_{n}
$$

Endow $G_{n}$ with the structure of a real nilpotent Lie-group by identifying it with the subgroup of $\mathrm{Gl}(2 n+2, \mathbb{C})$ consisting of upper triangular matrices of the form

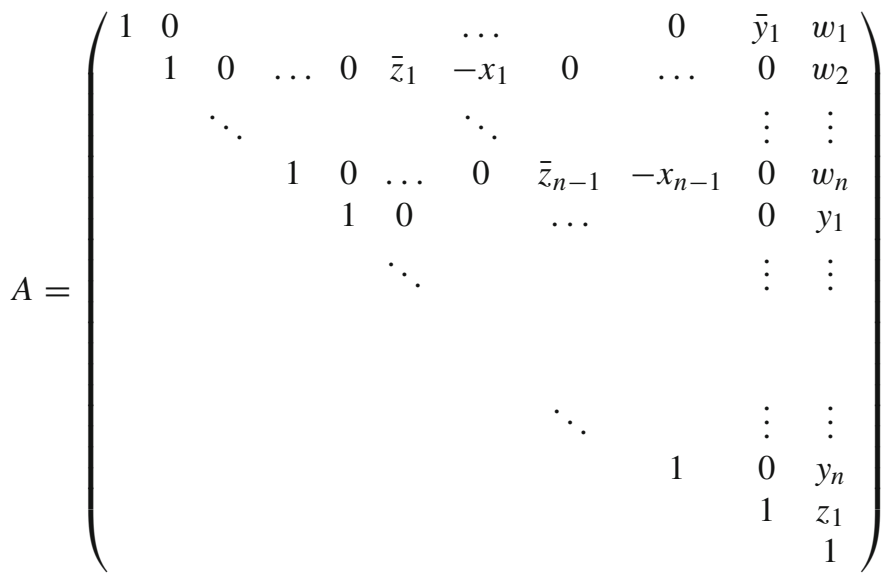

Let $\Gamma=G_{n} \cap \mathrm{Gl}(2 n+2, \mathbb{Z}[i])$, which is a lattice in the real Lie-group $G$. Note that if $g \in G_{n}$ is a fixed element then the action on the left, $g^{\prime} \mapsto g g^{\prime}$, is holomorphic with respect to the complex structure on $\mathbb{C}^{4 n-2}$. The quotient

$$
X_{n}=\Gamma / G_{n}
$$

is a compact complex manifold; more precisely, it is a compact nilmanifold with left-invariant complex structure.

Remark 1 The manifold $X_{n}$ admits a simple geometric description in terms of principal holomorphic torus bundles: the centre of $G_{n}$ is given by the matrices for which all $x_{i}, y_{i}$ and $z_{i}$ vanish and hence isomorphic (as a Lie group) to $\mathbb{C}^{n}$. This yields an exact sequence of real Lie-groups

$$
0 \rightarrow \mathbb{C}^{n} \rightarrow G_{n} \rightarrow \mathbb{C}^{3 n-2} \rightarrow 0
$$

which is compatible with the action of $\Gamma$. Denoting by $T_{k}$ the quotient $\mathbb{C}^{k} / \mathbb{Z}[i]^{k}$ the exact sequence induces a $T_{n}$ principal bundle structure on $X_{n} \rightarrow T_{3 n-2}$.

The space of left-invariant 1 -forms $U$ is spanned by the components of $A^{-1} d A$ and their complex conjugates, so a basis for the forms of type $(1,0)$ is given by

$$
d x_{1}, \ldots, d x_{n-1}, d y_{1}, \ldots, d y_{n}, d z_{1}, \ldots, d z_{n-1}, \omega_{1}, \ldots, \omega_{n}
$$


where

$$
\begin{aligned}
& \omega_{1}=d w_{1}-\bar{y}_{1} d z_{1}, \\
& \omega_{k}=d w_{k}-\bar{z}_{k-1} d y_{k-1}+x_{k-1} d y_{k} \quad(k=2, \ldots, n) .
\end{aligned}
$$

For later reference we calculate the differentials of the above basis vectors:

$$
\begin{array}{rlrl}
d\left(d x_{i}\right) & =d\left(d z_{i}\right)=0 & & (i=1 \cdots n-1) \\
d\left(d y_{i}\right) & =0 & & (i=1 \cdots n) \\
d \omega_{1} & =-d \bar{y}_{1} \wedge d z_{1} & \\
d \omega_{i} & =d x_{i-1} \wedge d y_{i}+d y_{i-1} \wedge d \bar{z}_{i-1} &
\end{array}
$$

The following lemma shows that the Frölicher spectral sequence of $X_{n}$ has nonvanishing differential $d_{n}$ thus proving our Theorem.

Lemma 1 The differential form $\beta_{1}=\bar{\omega}_{1} \wedge d \bar{z}_{2} \wedge \cdots \bar{d} z_{n-1}$ defines a class $\left[\beta_{1}\right]_{n} \in$ $E_{n}^{0, n-1}$ and

$$
d_{n}\left(\left[\beta_{1}\right]_{n}\right)=(-1)^{n-2}\left[d x_{1} \wedge \cdots \wedge d x_{n-1} \wedge d y_{n}\right]_{n} \neq 0 \text { in } E_{n}^{n, 0} .
$$

Proof By Remark 1 the projection to the $(x, y, z)$-coordinates endows $X_{n}$ with the structure of holomorphic principal torus bundle over a complex torus. By the results of [2] the inclusion of left-invariant forms into the double complex $\left(\mathcal{A}^{p, q}\left(X_{n}\right), \partial, \bar{\partial}\right)$ induces an isomorphism on the $E_{1}$-terms of the respective spectral sequences. Thus for our purpose we may work with left-invariant forms only, that is, start with the $E_{0}$-term

$$
E_{0}^{p, q}=\Lambda^{p} U \otimes \Lambda^{q} \bar{U}
$$

A $(p, q)$-form $\alpha$ lives to $E_{r}$ if it represents a class in $E_{r}^{p, q}$, which is a subquotient of $E_{0}^{p, q}$; the resulting class will be denoted by $[\alpha]_{r}$.

$\beta_{1}$ defines a class in $E_{n}$. As explained in $[1, \S 14, \mathrm{p} .161 \mathrm{ff}]$ this is equivalent to the existence of a zig-zag of length $n$, that is, a collection of elements $\beta_{2}, \ldots, \beta_{n}$ such that

$$
\beta_{i} \in E_{0}^{p+i, q-i}, \quad \bar{\partial} \beta_{1}=0, \quad \partial \beta_{i-1}+\bar{\partial} \beta_{i}=0 \quad(i=2, \ldots n)
$$

Consider the following differential forms $\beta_{k}$ of bidegree $(k-1, n-k)$ :

$$
\begin{aligned}
& \beta_{2}=\omega_{2} \wedge d \bar{z}_{2} \wedge \cdots \wedge \bar{z}_{n-1} \\
& \beta_{k}=d x_{1} \wedge \cdots \wedge d x_{k-2} \wedge \omega_{k} \wedge d \bar{z}_{k} \wedge \cdots \wedge \bar{z}_{n-1} \quad(3 \leq k \leq n-1) \\
& \beta_{n}=d x_{1} \wedge \cdots \wedge d x_{n-2} \wedge \omega_{n}
\end{aligned}
$$


A simple calculation shows that

$$
\begin{gathered}
\bar{\partial} \beta_{1}=0, \\
\partial \beta_{1}=-d y_{1} \wedge d \bar{z}_{1} \wedge \cdots \wedge d \bar{z}_{n-1}=-\bar{\partial} \beta_{2},
\end{gathered}
$$

and for $2 \leq k \leq n-1$

$$
\partial \beta_{k}=(-1)^{k-2} d x_{1} \wedge \cdots \wedge d x_{k-1} \wedge d y_{k} \wedge d \bar{z}_{k} \wedge \cdots \wedge d \bar{z}_{n-1}=-\bar{\partial} \beta_{k+1} \text {. }
$$

Therefore these elements define a zig-zag and $\beta_{1}$ defines a class in $E_{n}^{0, n-1}$.

It remains to prove that

$$
d_{n}\left[\beta_{1}\right]_{n}=\left[\partial \beta_{n}\right]_{n}=(-1)^{n-2}\left[d x_{1} \wedge \cdots \wedge d x_{n-1} \wedge d y_{n}\right]_{n}
$$

defines a non-zero class in $E_{n}^{n, 0}$, or equivalently, that $\beta_{1}$ does not live to $E_{n+1}$. In other words, we have to prove that does not exist a zig-zag of length $n+1$ for $\beta_{1}$. Since we are in a first quadrant double complex we have $E_{0}^{n,-1}=0$ and there exists a zig-zag of length $n+1$ if and only if there exists a zig-zag $\left(\beta_{1}, \beta_{2}^{\prime}, \ldots, \beta_{n}^{\prime}\right)$ of length $n$ such that $\partial \beta_{n}^{\prime}=0$.

To see that this cannot happen we put

$U_{1}=\left\langle d x_{1}, \ldots, d x_{n-1}, d y_{1}, \ldots, d y_{n}, d z_{1}, \ldots, d z_{n-1}\right\rangle_{\mathbb{C}}$ and $U_{2}=\left\langle\omega_{1}, \ldots, \omega_{n}\right\rangle_{\mathbb{C}}$

such that $U=U_{1} \oplus U_{2}$. The above basis of $U$ and its complex conjugate induce a basis on each exterior power and a decomposition

$$
\Lambda^{n}(U \oplus \bar{U})=\Lambda^{n}\left(U_{1} \oplus \bar{U}_{1}\right) \oplus S^{n},
$$

where $S^{n}$ is spanned by wedge products of basis elements, at least one of which is in $U_{2} \oplus \bar{U}_{2}$.

The elements $\beta_{k}$ and $(-1)^{k} \partial \beta_{k}$ are basis vectors and we decompose

$$
E_{0}^{k-1, n-k}=\beta_{k} \mathbb{C} \oplus V_{k} \quad \text { and } \quad E_{0}^{k, n-k}=\partial \beta_{k} \mathbb{C} \oplus W_{k},
$$

where $V_{k}$ (resp. $\left.W_{k}\right)$ is spanned by all other basis elements of type $(k-1, n-k)$ (resp. $(k, n-k)$ ). Let $\xi_{k}$ be the element of the dual basis such that $\left.\xi_{k}\right\lrcorner \partial \beta_{k}=1$ and the contraction with any other basis element is zero.

The differentials $\partial$ and $\bar{\partial}$ respect this decomposition, in the sense that

$$
\partial\left(V_{k}\right) \subset W_{k} \text { and } \bar{\partial}\left(V_{k}\right) \subset W_{k-1}
$$

More precisely, let $\alpha$ be one of the forms in our chosen basis for $\Lambda^{n-1}(U \oplus \bar{U})$. Recall that $\alpha$ is a decomposable form. Then

$$
\begin{aligned}
& \left.\partial \alpha \notin W_{k} \Longleftrightarrow \xi_{k}\right\lrcorner \partial \alpha \neq 0 \Longleftrightarrow \alpha=\beta_{k}, \\
& \left.\bar{\partial} \alpha \notin W_{k} \Longleftrightarrow \xi_{k}\right\lrcorner \bar{\partial} \alpha \neq 0 \Longleftrightarrow \alpha=\beta_{k+1},
\end{aligned}
$$


which implies (1). If $\alpha \in \Lambda^{n-1}\left(U_{1} \oplus \bar{U}_{1}\right)$ then $d \alpha=0$ and the claim is trivial. If the form $\alpha$ contains at least two basis elements of $U_{2} \oplus \bar{U}_{2}$ then each summand of $d \alpha$ with respect to the basis contains at least one element of $U_{2} \oplus \bar{U}_{2}$, in other words, $d \alpha \in S^{n}$. Since $\left.\xi_{k}\right\lrcorner S^{n}=0$ the claim is true also for those elements.

The remaining elements of the basis are of the form $\pm \alpha^{\prime} \wedge \omega_{i}$ or $\pm \alpha^{\prime} \wedge \bar{\omega}_{i}$ for some $\alpha^{\prime}$ in our chosen basis for $\Lambda^{n-2}\left(U_{1} \oplus \bar{U}_{1}\right)$. Paying special attention to the counting variable $d y_{k}$ this case is easily checked by looking for solutions of the equation

$$
\begin{aligned}
\partial\left(\alpha^{\prime} \wedge \omega_{i}\right) & =(-1)^{n-1} \alpha^{\prime} \wedge \partial \omega_{i} \\
& =(-1)^{k-2} d x_{1} \wedge \cdots \wedge d x_{k-1} \wedge d y_{k} \wedge d \bar{z}_{k} \wedge \cdots \wedge d \bar{z}_{n-1}=\partial \beta_{k},
\end{aligned}
$$

and similarly in the other cases involving either $\bar{\partial}$ or $\bar{\omega}_{i}$.

Thus if $\left(\beta_{1}, \beta_{2}^{\prime}, \ldots, \beta_{n}^{\prime}\right)$ is any zig-zag of length $n$ for $\beta_{1}$ then $\beta_{k}^{\prime} \equiv \beta_{k} \bmod V_{k}$ by (1) and, in particular,

$$
\partial \beta_{n}^{\prime} \equiv \partial \beta_{n} \not \equiv 0 \quad \bmod W_{n}
$$

Thus $\beta_{1}$ does not live to $E_{n+1}$ and $d_{n}\left[\beta_{1}\right]_{n}$ is non-zero as claimed.

Remark 2 In the original publication we constructed a compact complex manifold in a very similar way and an element $\left[\beta_{1}\right]_{n} \in E_{n}^{0, n-1}$. However, our claim that $d_{n}\left(\left[\beta_{1}\right]_{n}\right) \neq$ 0 was wrong: while the constructed zig-zag could not be extended, the sequence of elements (in the notation of [original publication, Lem. 2])

$$
\left(\beta_{1}, d x_{1} \wedge \bar{\omega}_{2} \wedge d \bar{x}_{3} \wedge \cdots \wedge d \bar{x}_{n-1}, 0,0, \ldots\right)
$$

gives an infinite zig-zag for $\beta_{1}$. In other words, the element considered gives an element of $E_{\infty}$ and thus a de Rham cohomology class.

Acknowledgments The second author would like to thank Daniele Grandini for pointing out the gap in the original publication and the first author for showing that the gap could not be filled and for joining the search for a correct example. Both authors were supported by the DFG via the second authors Emmy-Noether project and partially via SFB 701.

\section{References}

1. Bott, R., Tu, L.W.: Differential forms in algebraic topology. In: Graduate Texts in Mathematics, vol. 82. Springer, New York (1982)

2. Console, S., Fino, A.: Dolbeault cohomology of compact nilmanifolds. Transform. Groups 6(2), 111124 (2001) 